\title{
APPLICATION OF FINITE ELEMENT METHOD FOR ANALYSIS OF NANOSTRUCTURES
}

\author{
Jozef BOCKO*, Pavol LENGVARSKÝ \\ ${ }^{*}$ Faculty of Mechanical Engineering, Department of Applied Mechanics and Mechanical Engineering, \\ Technical University of Košice, Letná 9, 04200 Košice, Slovakia \\ jozef.bocko@tuke.sk, pavol.lengvarsky@tuke.sk
}

received 28 April 2016, revised 11 May 2017, accepted 15 May 2017

\begin{abstract}
The paper deals with application of the finite element method in modelling and simulation of nanostructures. The finite element model is based on beam elements with stiffness properties gained from the quantum mechanics and nonlinear spring elements with forcedisplacement relation are gained from Morse potential. Several basic mechanical properties of structures are computed by homogenization of nanostructure, e.g. Young's modulus, Poisson's ratio. The problems connecting with geometrical parameters of nanostructures are considered and their influences to resulting homogenized quantities are mentioned.
\end{abstract}

Key words: Beam Element, Spring Element, Young's Modulus, Poisson's Ratio

\section{INTRODUCTION}

The carbon structures attract the attention of researchers since the beginning of their investigation. Especially, electrical and mechanical properties of nanostructures have been widely investigated during last decade due to their interesting behaviour. The strength and stiffness characteristics of mechanical properties of nanostructures are much higher than those of steels. However, there is a problem with connection of carbon structure as a fibre with a steel matrix.

Nowadays, the analysis of nanostructure properties is based mostly on the following methods: molecular mechanics, molecular dynamics, continuum mechanics and experimental measurements. Tsai and Tu (2010) used molecular dynamics simulation for prediction of mechanical properties of graphene sheets. Continuum mechanics is well established for continuum theories of rods, beams, springs, shells, etc. (Li and Chou,2003; Meo and Rossi, 2006; Tserpes and Papanikos, 2005). The experimental measurements of mechanical properties of nanostructures are made in an atomic force microscope by nanoindentation (Lee et al., 2008).

The paper is devoted to investigation of Young's modulus and Poisson's ratio of nanostructure. These material characteristics are very important but still there are great uncertainties in their accurate determination. In the paper are investigated graphene sheets with different chiralities, widths, and lengths. The nanostructures are modelled using the MSM approach by either beam or spring elements. The carbon atoms are considered to be finite elements nodes and the interatomic interactions are represented by the structural elements (Li and Chou, 2003; Meo and Rossi, 2006; Hosseini and Moshrefzadeh, 2013).

\section{GRAPHENE SHEET IN MOLECULAR MECHANICS}

The graphene sheets can be regarded in molecular mechanics as large molecules consisting of carbon atoms and the atomic nuclei as material points. The motion of atomic nuclei is described by laws of force field. This field is expressed in the form of steric potential energy (Tserpes and Papanikos, 2005). The total steric potential energy (Saito et al., 1998; Thostenson et al., 2005) is a sum of energies due to valence or bonded interactions and nonbonded (van der Walls) interactions. It can be expressed by relation

$U_{\text {total }}=\sum U_{r}+\sum U_{\theta}+\sum U_{\phi}+\sum U_{\omega}+\sum U_{v d w}$,

where $U_{r}, U_{\theta}, U_{\phi}, U_{\omega}, U_{v d w}$ are a bond stretching, a bond angle bending, a dihedral angle torsion, an improper (out of plane) torsion and a non-bonded van der Waals interaction, respectively (Mayo et al., 1990; Shokrieh and Rafiee, 2010; Tserpes and Papanikos, 2005).

\subsection{Properties of beam element}

The properties of beam element are related to the first four terms of equation (1). By adopting the simplest harmonic forms and merging dihedral angle torsion and out-of-plane torsion into a single equivalent term, we can write the following relations

$U_{r}=\frac{1}{2} k_{r}\left(r-r_{0}\right)^{2}=\frac{1}{2} k_{r}(\Delta r)^{2}$,

$U_{\theta}=\frac{1}{2} k_{\theta}\left(\theta-\theta_{0}\right)^{2}=\frac{1}{2} k_{\theta}(\Delta \theta)^{2}$

$U_{\tau}=U_{\phi}+U_{\theta}=\frac{1}{2} k_{\tau}(\Delta \phi)^{2}$,

where $k_{r}, k_{\theta}, k_{\tau}, \Delta r, \Delta \theta, \Delta \phi$ are the bond stretching force constant, bond angle bending force constant, torsional resistance, the 
bond stretching increment, the bond angle variation and the angle variation of bond twisting, respectively (Brenner, 1990; Cornell et al., 1995; Rappe et al., 1992).

The elastic moduli of beam elements are evaluated from mechanical considerations - relations between the sectional stiffness parameters in structural mechanics and the force constants in molecular mechanics. In case the sections of beams representing carbon-carbon bonds, are assumed to be identical and circular, then the moments of inertia $\operatorname{are} \mathrm{I}_{\mathrm{x}}=\mathrm{I}_{\mathrm{y}}=\mathrm{I}$. Three stiffness parameters EA, EI and GJ are obtained from this assumption and from linkage among the energy terms in molecular mechanics and continuum mechanics. It is possible write relations

$U_{A}=\frac{1}{2} \int_{0}^{L} \frac{N^{2}}{E A} d L=\frac{1}{2} \frac{N^{2} L}{E A}=\frac{1}{2} \frac{E A}{L}(\Delta L)^{2}$,

$U_{M}=\frac{1}{2} \int_{0}^{L} \frac{M^{2}}{E I} d L=\frac{2 E I}{L} \alpha^{2}=\frac{1}{2} \frac{E I}{L}(2 \alpha)^{2}$,

$U_{T}=\frac{1}{2} \int_{0}^{L} \frac{T^{2}}{G J} d L=\frac{1}{2} \frac{T^{2} L}{G J}=\frac{1}{2} \frac{G J}{L}(\Delta \beta)^{2}$,

where $\mathrm{U}_{\mathrm{A}}$ is the strain energy of a uniform beam of length $\mathrm{L}$ subjected to axial force $\mathrm{N}, \Delta \mathrm{L}$ is axial elongation, $\mathrm{U}_{\mathrm{M}}$ is the strain energy of a uniform beam under bending moment $M, \alpha$ is the rotational angle at the ends of the beam, $U_{T}$ is the strain energy of a uniform beam under tension $\mathrm{T}$ and $\Delta \beta$ is the relative rotation between the ends of the beam ( $\mathrm{Li}$ and Chou, 2003; Tserpes and Papanikos, 2005).

Comparision of equations (2)-(4) and (5)-(7) leads to relations $\frac{E A}{L}=k_{r}, \frac{E I}{L}=k_{\theta}, \frac{G J}{L}=k_{\tau}$.

On the basis of these equations a beam element (Fig. 1) is created and its elastic properties for further computations are

$d=4 \sqrt[4]{\frac{k_{\theta}}{k_{r}}}, E=\frac{k_{r}{ }^{2} L}{4 \pi k_{\theta}}, G=\frac{k_{r}{ }^{2} k_{\tau} L}{8 \pi k_{\theta}^{2}}$,

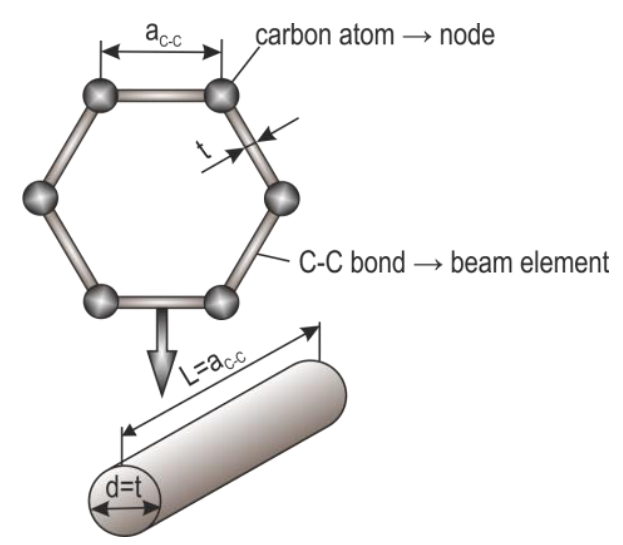

Fig. 1. Hexagon unit cell of graphene sheet with beam elements

where constants $\mathrm{k}_{\mathrm{r}}, \mathrm{k}_{\theta}, \mathrm{k}_{\tau}, \mathrm{L}$ are $\mathrm{k}_{\mathrm{r}}=6.52 \times 10^{-7} \mathrm{Nnm}^{-1}$, $\mathrm{k}_{\theta}=8.76 \times 10^{-10} \mathrm{~N} \mathrm{~nm} \mathrm{rad}{ }^{-1}, \mathrm{k}_{\tau}=2.78 \times$

$10^{-7} \mathrm{~N} \mathrm{n} \mathrm{m} \mathrm{rad}{ }^{-2}$ and $\mathrm{L}=\mathrm{a}_{\mathrm{C}-\mathrm{C}}=0.1421 \mathrm{~nm}$ (Marenić et al., 2013).

Accordingly, the quantities from equations (9) are calculated and we come to diameter $\mathrm{d}=0.147 \mathrm{~nm}$, elastic modulus $\mathrm{E}=5.4875 \mathrm{TPa}$ and $\mathrm{G}=0.871 \mathrm{TPa}$ for beam elements.

\subsection{Properties of spring elements}

The properties of spring elements come from the Morse potential. The Morse potential is used for evaluation of interactions of $\mathrm{C}-\mathrm{C}$ bond stretching and $\mathrm{C}-\mathrm{C}-\mathrm{C}$ bond angle bending. The Morse potential for $\mathrm{C}-\mathrm{C}$ and $\mathrm{C}-\mathrm{C}-\mathrm{C}$ bonds is computed according to eqs. (10)-(11). All necessary parameters for computations are given in Tab. 1 (Machida, 1999; Rafiee and Heidarhaei, 2012).

$u_{r}=D_{e}\left\{\left[1-e^{-\beta\left(r-r_{0}\right)}\right]^{2}\right\}$

$u_{\theta}=\frac{1}{2} k_{\theta}\left(\theta-\theta_{0}\right)^{2}\left[1+k_{\text {sextic }}\left(\theta-\theta_{0}\right)^{4}\right]$.

In this paper $\mathrm{C}-\mathrm{C}$ and $\mathrm{C}-\mathrm{C}-\mathrm{C}$ bonds are modelled with nonlinear spring elements. The knowledge of force-displacement relationship for $\mathrm{C}-\mathrm{C}$ bonds is necessary and this quantity is derived from the Morse potential using eq. (10) (Rafiee and Heidarhaei, 2012)

$F\left(r-r_{0}\right)=2 \beta D_{e}\left[1-e^{-\beta\left(r-r_{0}\right)}\right] e^{-\beta\left(r-r_{0}\right)}$.

Function for $\mathrm{C}-\mathrm{C}-\mathrm{C}$ bonds is derived by using eq. (11) and the result is expressed by equation (Rafiee and Heidarhaei, 2012]

$M\left(\theta-\theta_{0}\right)=k_{\theta}\left(\theta-\theta_{0}\right)\left[1+3 k_{\text {sextic }}\left(\theta-\theta_{0}\right)^{4}\right]$.

Tab. 1. Parameters for the Morse potential

\begin{tabular}{|c|c|}
\hline parameter & value \\
\hline$D_{e}$ & $6.03105 \times 10^{-19} \mathrm{Nm}$ \\
\hline$\beta$ & $2.6259 \times 10^{10} \mathrm{~m}^{-1}$ \\
\hline$r_{0}$ & $0.1421 \mathrm{~nm}$ \\
\hline$k_{\theta}$ & $0.9 \times 10^{-18} \mathrm{~N} \frac{\mathrm{m}}{\mathrm{rad}^{2}}$ \\
\hline$k_{\text {sextic }}$ & $0.754 \mathrm{rad}^{4}$ \\
\hline$\theta_{\theta}$ & $2.094 \mathrm{rad}$ \\
\hline
\end{tabular}

Every hexagon of graphene sheet is modelled by 12 nonlinear springs. Six outer springs represent bond stretching and six inner springs represent bond angle bending (Fig. 2). Bond angle torsion is not considered in the frame of this spring model.

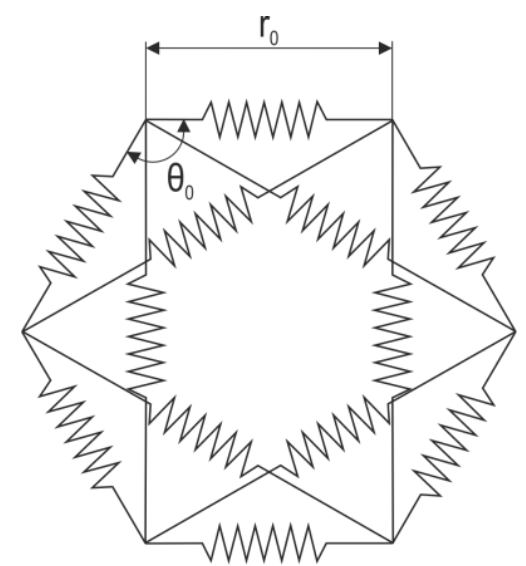

Fig. 2. Hexagon unit cell of graphene sheet with 12 nonlinear spring elements

The change of length of nonlinear spring of $\mathrm{C}-\mathrm{C}-\mathrm{C}$ bond leads to change of bond angle. This fact can be described by equation 
$\Delta \theta=\frac{2(\Delta R)}{r_{0}}$

where $\Delta \theta$ is variation of bond angle, $r_{0}$ is $\mathrm{C}-\mathrm{C}$ bond length and $\Delta \mathrm{R}$ is change of spring element length. Accordingly, forcedisplacement relationship in eq. (13) is rewritten into form of relation

$F_{\left(R-R_{0}\right)}=\frac{4}{r_{0}^{2}} k_{\theta}\left(R-R_{0}\right)\left[1+\frac{48}{r_{0}^{4}} k_{\text {sextic }}\left(R-R_{0}\right)^{4}\right]$.

The nonlinear behaviour of this spring is shown in Fig. 3 and Fig. 4.

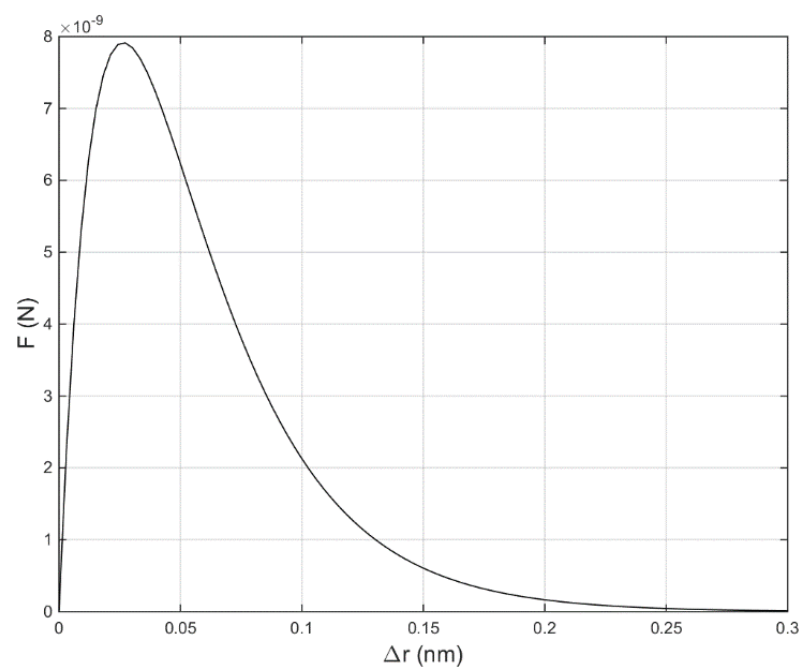

Fig. 3. Force-displation curve for nonlinear spring representing bond stretching

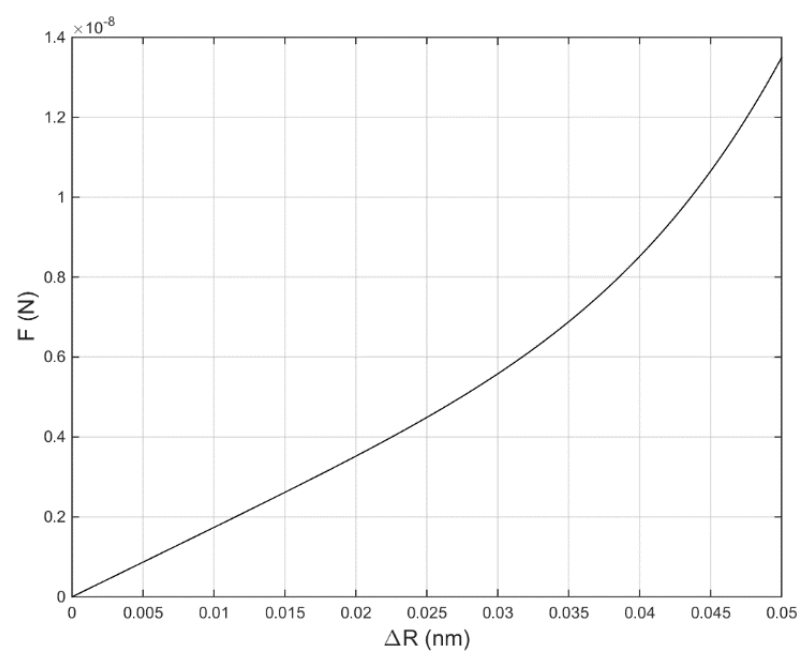

Fig. 4. Force-displation curve for nonlinear spring representing change of bond angle

\section{NUMERICAL RESULTS}

The numerical computations are accomplished by the finite element program Ansys. The structural response of the graphene sheets under axial loads is analyzed for both model types (Figs. 56 ). Carbon atoms are considered to be nodes in structure and the nodes are joined by beam elements or nonlinear spring elements. To simulate the uniaxial load, one side of the graphene sheet is restrained in all direction. The graphene sheets are stretched by applying an axial displacement on the opposite side. From these simulations the Young's modulus $E$, the Poisson's ratio $\mu$ of graphene sheets are obtained. The two graphene sheets with armchair chirality $(6,6)$ and zigzag chirality $(10,0)$ are modelled and analyzed. The width of the graphene sheet $(6,6)$ and $(10,0)$ is $2.5578 \mathrm{~nm}$ and $2.4612 \mathrm{~nm}$, respectively. The length of the graphene sheets varied in interval $3 \mathrm{~nm}$ to $100 \mathrm{~nm}$.

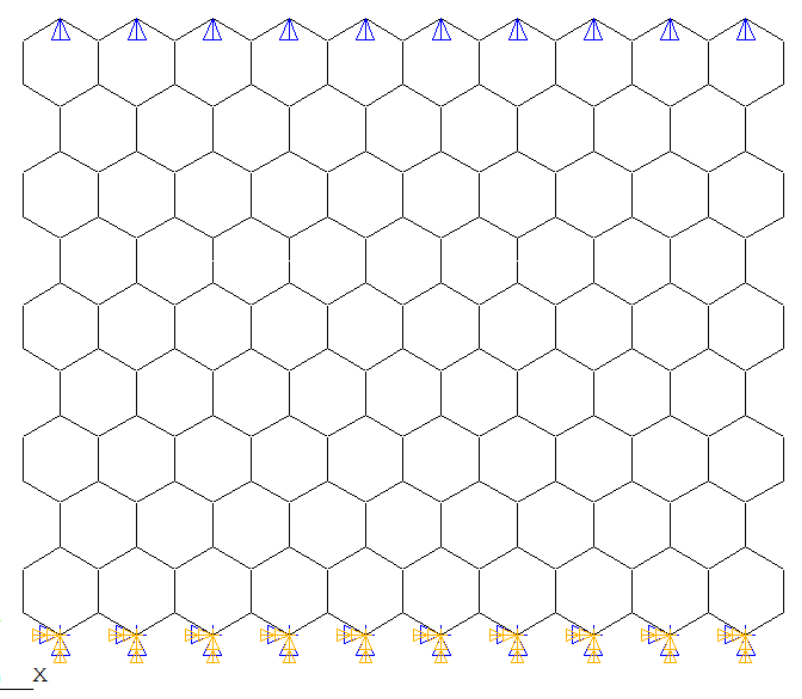

Fig. 5. Graphene sheet with beam elements and boundary conditions

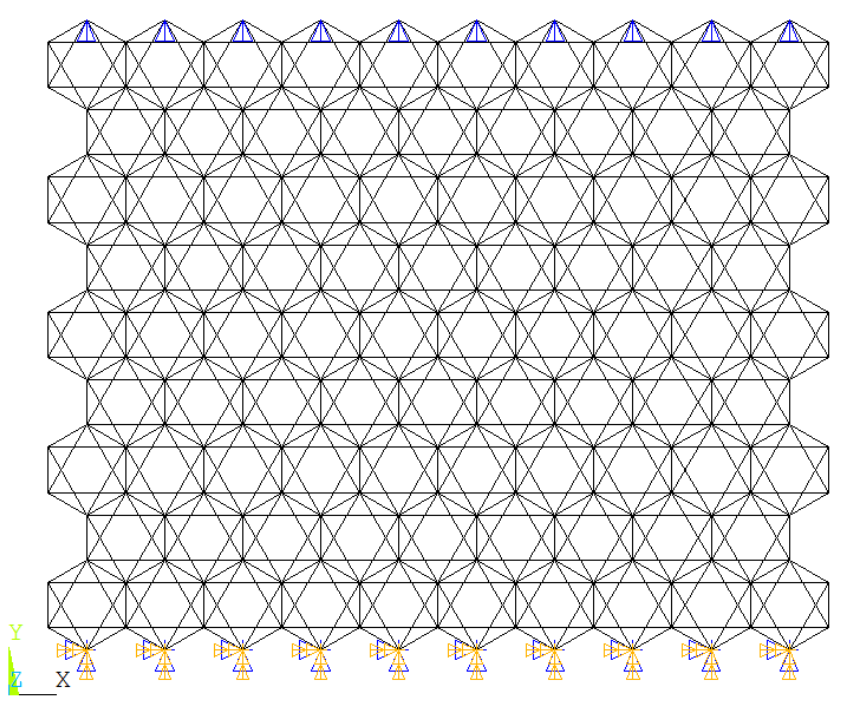

Fig. 6. Graphene sheet with nonlinear spring elements and boundary conditions

Young's moduls $E$ is calculted from equation

$E=\frac{\sigma}{\varepsilon}=\frac{F / W t}{\Delta L / L}$

where $\mathrm{F}$ is reaction force, $\mathrm{W}$ is width, $\mathrm{t}$ is thickness, $\Delta \mathrm{L}$ is elongation and $\mathrm{L}$ is length of graphene sheet. The thickness of graphene sheet is assumed to be $0.34 \mathrm{~nm}$. Poisson's ratio is computed from equation

$\mu=\frac{\Delta W / W}{\Delta L / L}$

where $\Delta \mathrm{W}$ is constriction of graphene sheet. 
All computed values of Young's moduls E and the Poisson's ratio $\mu$ are shown in Figs. 7-8.

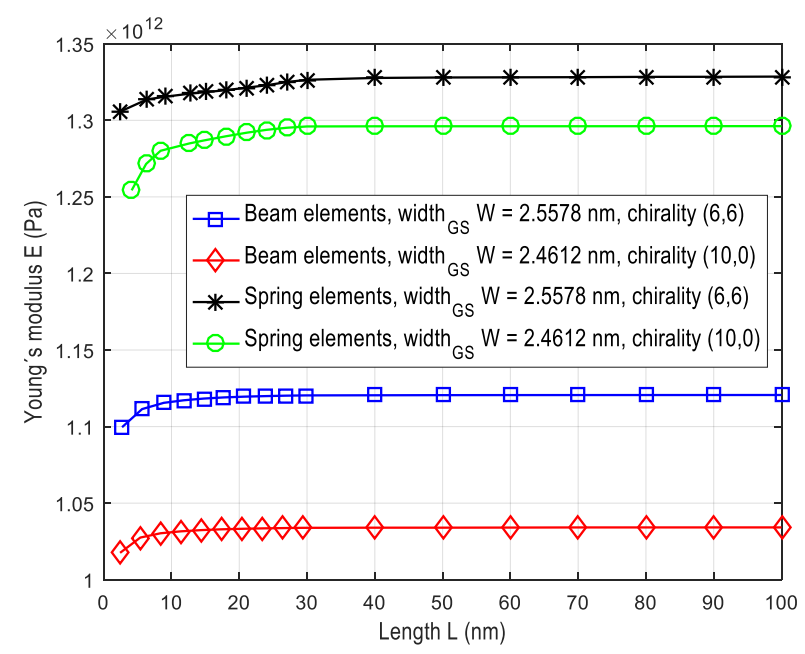

Fig. 7. Young's modulus of graphene sheet with diferent elements, length and width

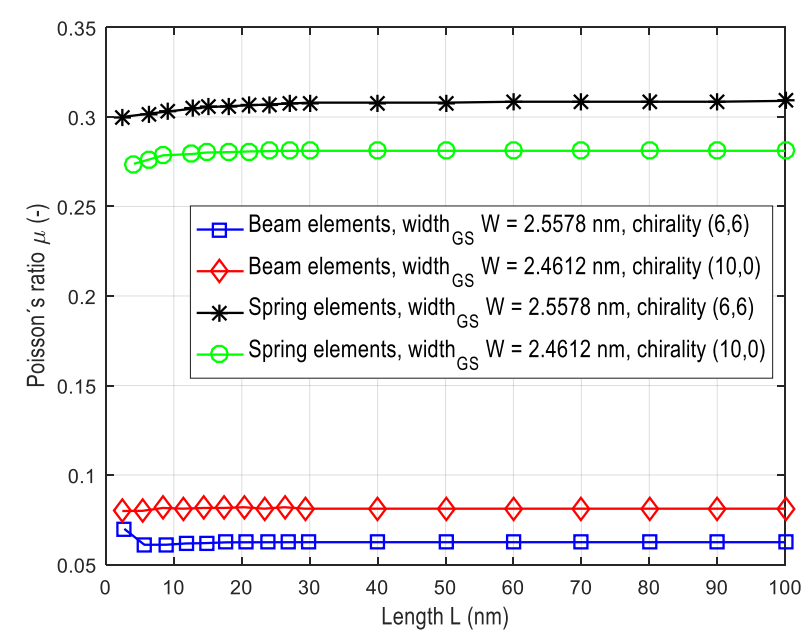

Fig. 8. Poisson's ratio of graphene sheet with diferent elements, length and width

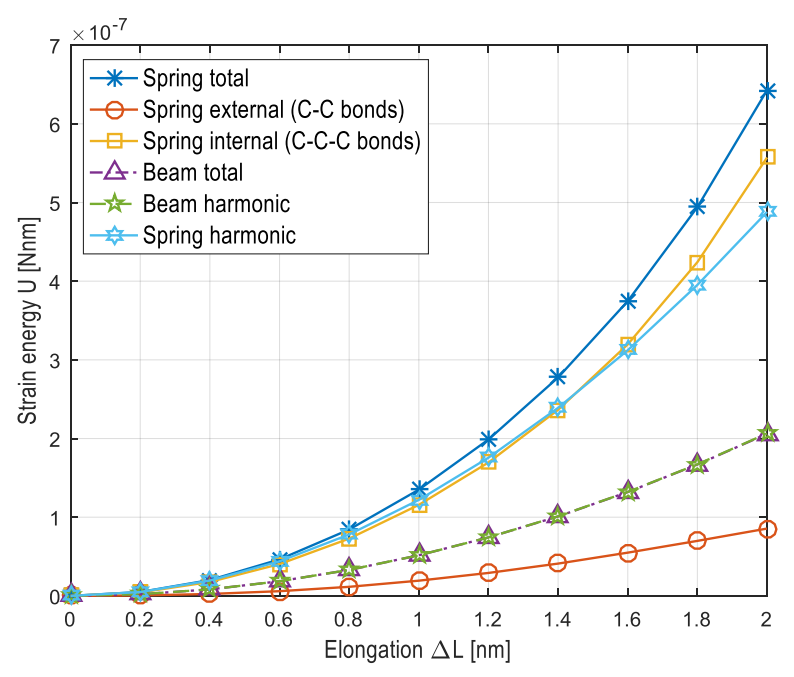

Fig. 9. Strain energy of graphene sheet with dimensions $2.4612 x$ $8.3858 \mathrm{~nm}$ subjected to axial tension obtained from beam and spring model

\section{CONCLUSION}

The Young's modulus $E$ increases slightly with the size of the sheets and depends on the chirality. The beam and spring models give almost the same results for $E$ with the obtained values being in good agreement with literature (Hartmann et al., 2013; Meo and Rossi, 2006; Rafiee and Heidarhaei, 2012; Scarapa et al., 2009; Tserpes and Papanikos, 2005). For the Poisson's ratio $\mu$ the beam and spring models give strongly different results. Depending on the chirality, values of $\mu$ between 0.08 and 0.06 are obtained for the beam model. For the spring model values of $\mu$ vary between 0.28 and 0.31 and accordingly there are no strong size and chirality dependencies for the same model. Although the beam and spring models give different results for $\mu$ all values are within the range reported in literature (Hartmann et al., 2013; Hemmasizadeh et al., 2008; Ru, 2000; Sakhaee-Pour, 2009; Tsai and $\mathrm{Tu}, 2010)$. It is assumed that different values of $\mu$ depend on the different representation of the bond angle bending in the beam and spring models. In order to see the influence of the bond angle bending, the total strain energy of the stretched graphene sheets is estimated for both models. The results from numerical computations are shown in Fig. 9. For the spring model the total strain energy can be split into a bond stretching and a bond angle contribution. It can be seen that the total strain energy of the spring model is much higher than the strain energy of the beam model, where stronger contribution comes from the bond angle bending deformations. This shows the bond angle bending is probably not correctly represented in the beam model. This may lead to the relatively small Poisson's ratio of this model and may cause further issues for loading cases where bond angle bending is important. By comparison between strain energy of the spring model and corresponding harmonic potentials it is also shown that graphene sheets work in linear elastic area up to a stretch of approximately 10 percent. Due to the assumptions made for the beam elements no non-linear behavior can be observed for this model. Hence, it is valid only for small deformations.

\section{REFERENCES}

1. Brenner D.W. (1990), Empirical potential for hydrocarbons for use in simulating the chemical vapor deposition of diamond films, Physical Review B, Vol. 42, 9458.

2. Cornell W.D., Cieplak P., Bayly C.I. (1995), A second generation force-field for the simulation of proteins, nucleic-acids, and organicmolecules, Journal of American Chemical Society, 117, 5179-5197.

3. Hartmann M.A., Todt M., Rammerstorfer F.G., Fisher F.D., Paris O. (2013), Elastic properties of graphene obtained by computational mechanical tests, Europhysics Letters, 103, 68004-p1-68004-p6.

4. Hemmasizadeh A., Mahzoon M., Hadi E., Khandan R. (2008), A method for developing the equivalent continuum model of a single layer graphene sheet, Thin Solid Films, 516, 7636-7640.

5. Hosseini K.S.A., Moshrefzadeh S.H. (2013), Mechanical properties of double-layered graphene sheets, Computational Materials Science, 69, 335-343.

6. Lee C., Wei X., Kysar J.W., Hone J. (2008), Measurement of the elastic properties and intrisic strength of monolayer graphene, Science, 321, 385-388.

7. Li Ch., Chou T.W. (2003), A structural mechanics approach for the analysis of carbon nanotube, International Journal of Solids and Structures, 40, 2487-2499.

8. Machida K. (1999), Principles of Molecular Mechanics, Kodansha and John Wiley \& Sons Co-publication, Tokyo. 
16. Sakhaee-Pour A. (2009), Elastic properties of single-layered graphene sheet, Solid State Communications, 149, 91-95.

17. Scarapa F., Adhikari S., Srikantha P. (2009), Effective elastic mechanical properties of single layer graphene sheets, Nanotechnology, 20, 065709.

18. Shokrieh M.M, Rafiee R. (2010) Prediction of Young's modulus of graphene sheets and carbon nanotubes using nanoscale continuum mechanics approach, Materials \& Design, 31, 790-795.

19. Thostenson E.T., Chunyu L., Chou T.W. (2005), Nanocomposites in context, Composite Science and Technology, 65, 491-516.

20. Tsai J.L., Tu J.F. (2010), Characterizing mechanical properties of graphite using molecular dynamics simulation, Materials \& Design, 31, 194-199.

21. Tserpes K.I., Papanikos P. (2005), Finite element modelling of single-walled carbon nanotubes, Composites Part B, 36, 468-477.

This work was supported by grants from the Slovak Grant Agency VEGA no. $1 / 0731 / 16$. 